\title{
High degeneracy of effective Hamiltonian in two dimensions
}

\author{
Yifeng Yu
}

Abstract. Consider the effective Hamiltonian $\bar{H}(p)$ associated with the mechanical Hamiltonian $H(p, x)=\frac{1}{2}|p|^{2}+V(x)$. We prove that for generic $V, \bar{H}$ is piecewise one-dimensional in a dense open set in two dimensions using Aubry-Mather theory.

\section{Introduction}

Assume that $H=H(p, x) \in C\left(\mathbb{R}^{n} \times \mathbb{R}^{n}\right)$ is $\mathbb{Z}^{n}$-periodic in $x$ and uniformly coercive in $p$, i.e.,

$$
\lim _{|p| \rightarrow+\infty} \min _{x \in \mathbb{R}^{n}} H(p, x)=+\infty .
$$

For each $\varepsilon>0$, let $u^{\varepsilon} \in C\left(\mathbb{R}^{n} \times[0, \infty)\right)$ be the viscosity solution to the Hamilton-Jacobi equation

$$
\begin{cases}u_{t}^{\varepsilon}+H\left(D u^{\varepsilon}, \frac{x}{\varepsilon}\right)=0 & \text { in } \mathbb{R}^{n} \times(0, \infty), \\ u^{\varepsilon}(x, 0)=g(x) & \text { on } \mathbb{R}^{n} .\end{cases}
$$

It was proved by Lions, Papanicolaou and Varadhan ([13]) that $u^{\varepsilon}$, as $\varepsilon \rightarrow 0$, converges locally uniformly to $u$, the solution of the effective equation

$$
\begin{cases}u_{t}+\bar{H}(D u)=0 & \text { in } \mathbb{R}^{n} \times(0, \infty), \\ u(x, 0)=g(x) & \text { on } \mathbb{R}^{n} .\end{cases}
$$

Here $\bar{H}: \mathbb{R}^{n} \rightarrow \mathbb{R}$ is the so-called effective Hamiltonian, which is determined by the following cell problem: for any $p \in \mathbb{R}^{n}$, there exists a unique number $\bar{H}(p) \in \mathbb{R}$ such that the equation

$$
H(p+D v, x)=\bar{H}(p)
$$

has a periodic viscosity solution. When $H$ is convex in $p$, the effective Hamiltonian is convex and has a variational formulation

$$
\bar{H}(p)=\inf _{\phi \in C^{1}\left(\mathbb{T}^{n}\right)} \max _{x \in \mathbb{T}^{n}} H(p+D \phi(x), x) .
$$

2020 Mathematics Subject Classification. 35B27, 35F20, 37J51.

Keywords. Homogenization, effective Hamiltonian, Aubry-Mather theory, weak KAM theory. 
Although there is a lot of literature regarding homogenization of Hamilton-Jacobi equations in various settings, not much is known about the finer properties of the effective Hamiltonian $\bar{H}$ due to a lack of tools. In this paper, we focus on the mechanical Hamiltonian

$$
H(p, x)=\frac{1}{2}|p|^{2}+V(x) .
$$

Here $V$ is assumed to be $C^{k}\left(\mathbb{R}^{n}\right)$ for $k \geq 2$ and $\mathbb{Z}^{n}$-periodic. The following properties hold in any dimension.

- Property 1: Quadratic growth.

$$
\frac{1}{2}|p|^{2}+\min _{\mathbb{R}^{n}} V \leq \bar{H}(p) \leq \frac{1}{2}|p|^{2}+\max _{\mathbb{R}^{n}} V .
$$

- Property 2: Minimum value.

$$
\min _{\mathbb{R}^{n}} \bar{H}=\max _{\mathbb{R}^{n}} V
$$

More interestingly, for quite general $V$, the minimum level set

$$
F_{0}=\left\{p \in \mathbb{R}^{n} \mid \bar{H}=\max _{\mathbb{R}^{n}} V\right\}
$$

is an $n$-dimensional convex set ([6]).

- Property 3: Strict convexity along any non-tangential direction. Using techniques from weak KAM theory, it was proved in [8] that $\bar{H}$ is not linear along any direction that is not tangent to its level set. In particular, this implies that if

$$
\bar{H}\left(\frac{p_{1}+p_{2}}{2}\right)=\frac{1}{2} \bar{H}\left(p_{1}\right)+\frac{1}{2} \bar{H}\left(p_{2}\right)
$$

then

$$
H\left(\lambda p_{1}+(1-\lambda) p_{2}\right)=\bar{H}\left(p_{1}\right) \text { for all } \lambda \in[0,1] .
$$

Although $\bar{H}$ inherits some global features of $\frac{1}{2}|p|^{2}$, its local properties could be drastically different from those of $\frac{1}{2}|p|^{2}$. In this paper, we will prove that for generic $V, \bar{H}$ is piecewise one-dimensional on a dense open set using Aubry-Mather theory. Precisely speaking, we have the following theorem:

Theorem 1.1. For any $k \geq 2$, there is a residual subset $\mathcal{E}$ of $C^{k}\left(\mathbb{T}^{2}\right)$ such that for every $V \in \mathcal{E}$, there exists a sequence of bounded open sets $\left\{O_{i}\right\}_{i \geq 1}$ in $\mathbb{R}^{2}$ such that

(1) the set

$$
O_{V}=\bigcup_{i=1}^{\infty} O_{i}
$$

is a dense open set in $\mathbb{R}^{2}$; 
(2) for each $i \in \mathbb{N}$, there exist a unit vector $q_{i} \in \mathbb{R}^{2}$ and a convex function $f_{i}: \mathbb{R} \rightarrow \mathbb{R}$ such that

$$
\bar{H}_{V}(p)=f_{i}\left(q_{i} \cdot p\right) \text { in } O_{i},
$$

where $\bar{H}_{V}$ is the effective Hamiltonian associated with $\frac{1}{2}|p|^{2}+V$.

In general, the above conclusion might not be true. For example, consider the separable case when $V(x)=h\left(x_{1}\right)+g\left(x_{2}\right)$. In dynamical systems, some generic perturbation mechanisms based on the Baire property in general topology have often been employed to filter out those exceptional situations. However, it is usually impossible to tell whether a concrete example is generic or not.

In the above theorem, $\mathcal{E}$ is the intersection of a sequence of dense open sets of $C^{k}\left(\mathbb{T}^{2}\right)$. There are two choices of $\mathcal{E}$. One choice is to directly use the residual set in [3, Cor. 1.2], whose existence is established under certain abstract frameworks of convex analysis. The other choice is the $\mathscr{E}$ constructed in the appendix, (A.1), which is weaker in the sense of dynamical systems but is more explicit and enough for our purpose. Moreover, the result is expected to hold for more general Hamiltonians. In this paper, for clarity of presentation we will focus only on the mechanical Hamiltonian, which is interesting enough.

\section{Notation and terminology}

- $\quad \mathbb{T}^{n}=\mathbb{R}^{n} / \mathbb{Z}^{n}$ represents the $n$-dimensional flat torus; $C^{k}\left(\mathbb{T}^{n}\right)$ is the set of all $\mathbb{Z}^{n}$ periodic $C^{k}\left(\mathbb{R}^{n}\right)$ functions.

- A vector $q \in \mathbb{R}^{n}$ is called a rational vector if there exists $\lambda \in \mathbb{R} \backslash\{0\}$ such that $\lambda q \in \mathbb{Z}^{n}$.

- $\quad(m, n) \in \mathbb{Z}^{2}$ is called irreducible if $|m|$ and $|n|$ are relatively prime.

- A curve $\xi: \mathbb{R} \rightarrow \mathbb{T}^{n}$ is called periodic if there exists $T>0$ such that

$$
\xi(t+T)=\xi(t) \quad \text { for all } t \in \mathbb{R},
$$

where $T$ is called a period. If $T_{0}>0$ is the minimal period of $\xi$ and $\xi$ is lifted to $\mathbb{R}^{n}$, then

$$
\xi\left(T_{0}\right)-\xi(0)=(m, n) \in \mathbb{Z}^{2}
$$

is the first homology class of $\xi$.

- Denote by $L(q, x): \mathbb{R}^{n} \times \mathbb{R}^{n} \rightarrow \mathbb{R}$ the Lagrangian

$$
L(q, x)=\sup _{p \in \mathbb{R}^{n}}\{q \cdot p-H(p, x)\}
$$

- $\quad$ For $p_{1}, p_{2} \in \mathbb{R}^{n}$, let

$$
\left[p_{1}, p_{2}\right]=\left\{t p_{1}+(1-t) p_{2} \mid t \in[0,1]\right\}
$$

be the line segment connecting $p_{1}$ and $p_{2}$. 


\section{Preliminaries}

For the readers' convenience, in this section we give a brief review about some basic knowledge and relevant results of Aubry-Mather theory and weak KAM theory. See [1,7-9] for more details. Our presentation will be mainly from the PDE point of view. Many parts are very close to the standard theory of Hamilton-Jacobi equations. The major difference lies in classical Aubry-Mather theory, which is based on two-dimensional topology and cannot be captured by PDE approaches.

Let $\mathbb{T}^{n}=\mathbb{R}^{n} / \mathbb{Z}^{n}$ be the $n$-dimensional flat torus and $H(p, x) \in C^{2}\left(\mathbb{R}^{n} \times \mathbb{R}^{n}\right)$ be a Hamiltonian satisfying

(H1) (periodicity) $x \mapsto H(p, x)$ is $\mathbb{Z}^{n}$-periodic;

(H2) (uniform convexity) there exists $\theta>0$ such that for all $\eta=\left(\eta_{1}, \ldots, \eta_{n}\right) \in \mathbb{R}^{n}$, and $(p, x) \in \mathbb{R}^{n} \times \mathbb{R}^{n}$,

$$
\sum_{i, j=1}^{n} \eta_{i} \frac{\partial^{2} H}{\partial p_{i} \partial p_{j}} \eta_{j} \geq \theta|\eta|^{2}
$$

A major goal in dynamical systems is to understand the long time behaviors of trajectories of the Hamiltonian system

$$
\left\{\begin{array}{l}
\dot{x}(t)=D_{p} H(p, x), \\
\dot{p}(t)=-D_{x} H(p, x) .
\end{array}\right.
$$

When the Hamiltonian $H$ is a small perturbation of the integrable case, the famous KAM theory based on analytic approaches says that most trajectories lie on invariant tori and hence are integrable. For general $H$, when $n=2$ classical Aubry-Mather theory provides a nice description of structures of action minimizing trajectories based on topological approaches. See Section 2.3 for more details. In [14], Mather extended Aubry-Mather theory to higher dimensions through variational methods. The weak KAM theory reveals interesting connections between Mather's theory and solutions to the cell problem (1.3). In fact, in the language of PDEs, the classical KAM theory can be formulated as follows: in the perturbative case, for "most" $Q=D \bar{H}(p)$, the cell problem has a unique smooth solution $v$ up to a constant and the corresponding invariant torus is given by

$$
\mathbb{T}_{Q}=\left\{(q, x) \in \mathbb{R}^{n} \times \mathbb{R}^{n}: p+D v(x)=D_{q} L(q, x)\right\} .
$$

\subsection{Aubry set and Mané set}

Let $v$ be a solution to the cell problem (1.3). We say that a curve $\gamma: \mathbb{R} \rightarrow \mathbb{R}^{n}$ is a global characteristic associated with $v$ if for all $t_{1}<t_{2}$ and $u(x)=p \cdot x+v$,

$$
\int_{t_{1}}^{t_{2}} L(\dot{\gamma}, \gamma)+\bar{H}(p) d s=u\left(\gamma\left(t_{2}\right)\right)-u\left(\gamma\left(t_{1}\right)\right) .
$$


Write

$$
J_{v}=\bigcup_{\gamma}\{(\dot{\gamma}(t), \gamma(t)) \mid t \in \mathbb{R}, \gamma \text { is a global characteristic of } v\}
$$

Such a $\gamma$ is also called a $(v, L, \bar{H}(p))$-calibrated curve in [9]. According to classical theory in Hamilton-Jacobi equations ([12]), saying that $\gamma$ is a global characteristic of $v$ is equivalent to saying that $v$ is differentiable along $\gamma$ and for all $t \in \mathbb{R}$,

$$
p+D v(\gamma(t))=D u(\gamma(t))=D_{q} L(\dot{\gamma}(t), \gamma(t)) \text {. }
$$

Moreover, owing to Lemma 2.1, every global characteristic is an absolutely minimizing curve with respect to $L(q, x)+\bar{H}(p)$. So it satisfies the Euler-Lagrange equation

$$
\frac{d\left(D_{q} L(\dot{\gamma}(t), \gamma(t))\right)}{d t}=D_{x} L(\dot{\gamma}(t), \gamma(t))
$$

A curve $\gamma: \mathbb{R} \rightarrow \mathbb{R}^{n}$ is called an absolutely minimizing curve with respect to $L(q, x)+c$ if for any $-\infty<s_{2}<s_{1}<\infty,-\infty<t_{2}<t_{1}<\infty$ and $\eta \in \operatorname{AC}\left(\left[s_{1}, s_{2}\right], \mathbb{R}^{n}\right)$ subject to $\eta\left(s_{2}\right)=\gamma\left(t_{2}\right)$ and $\eta\left(s_{1}\right)=\gamma\left(t_{1}\right)$ the following inequality holds:

$$
\int_{s_{1}}^{s_{2}}(L(\dot{\eta}(s), \eta(s))+c) d s \geq \int_{t_{1}}^{t_{2}}(L(\dot{\gamma}(s), \gamma(s))+c) d s .
$$

See Figure 1. Here $\operatorname{AC}([a, b], S)$ stands for the set of absolutely continuous curves $[a, b] \rightarrow S$.

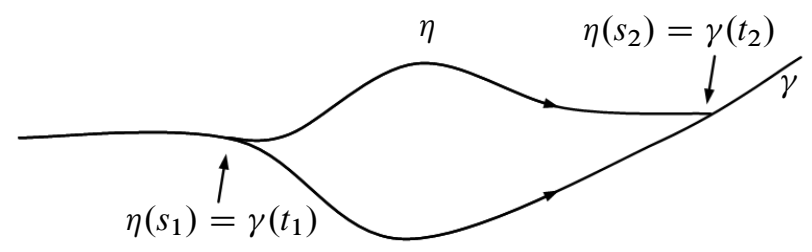

Figure 1

The curve $\gamma: \mathbb{R} \rightarrow \mathbb{R}^{n}$ is called a universal global characteristic if it is a characteristic for every viscosity solution to (1.3).

For $p \in \mathbb{R}^{n}$, the collection of all universal characteristics

$$
\tilde{\mathcal{A}}_{p}=\bigcup\{(\dot{\gamma}(t), \gamma(t)) \mid t \in \mathbb{R}, \gamma \text { is a universal global characteristic }\}=\bigcap_{\substack{v \text { is alution } \\ \text { to }(1.3)}} J_{v}
$$

is defined as the Aubry set. Hence the following graph property holds:

$$
\tilde{\mathcal{A}}_{p} \subset\left\{(q, x) \in \mathbb{R}^{n} \times \mathbb{R}^{n}: D v(x) \text { exists and } p+D v(x)=D_{q} L(q, x)\right\} .
$$

Write $\mathscr{A}_{p}$ as the projection of $\tilde{\mathscr{A}}_{p}$ on $\mathbb{R}^{n}$. 
Also, we define the collection of all global characteristics associated with viscosity solutions of (1.3),

$$
\tilde{\mathcal{N}}_{p}=\bigcup\{(\dot{\gamma}(t), \gamma(t)) \mid t \in \mathbb{R}, \gamma \text { is a global characteristic }\}=\bigcup_{\substack{v \text { is a solution } \\ \text { to (1.3) }}} J_{v}
$$

as the Mané set.

In standard definitions ([9]), the Aubry set and Mané set are on $\mathbb{R}^{n} \times \mathbb{T}^{n}$. Here for convenience, we lift them to $\mathbb{R}^{n} \times \mathbb{R}^{n}$. Let us highlight several key properties

- Property 4. Two absolutely minimizing curves cannot intersect twice unless they are the same after suitable translations in time. This property together with twodimensional topology plays a crucial role in classical Aubry-Mather theory in two dimensions.

- Property 5. Two universal global characteristics cannot intersect unless they are the same after suitable translation in $t$.

- Property 6. Any global characteristic, when it is projected to $\mathbb{T}^{n}$, cannot intersect itself unless the orbit is periodic.

- Property 7. If $\xi$ is a global characteristic, then for any sequence $T_{m} \rightarrow \infty$ as $m \rightarrow+\infty$,

$$
\lim _{m \rightarrow+\infty} \frac{\xi\left(T_{m}\right)-\xi(0)}{T_{m}} \in \partial \bar{H}(p)
$$

if the limit exists. Here $\partial \bar{H}(p)$ is the subdifferential of $\bar{H}$ at $p$. The full limit

$$
\lim _{T \rightarrow \infty} \frac{\xi(T)-\xi(0)}{T}
$$

if it exists, is called the rotation vector of $\xi$.

\subsection{Mather set}

Denote by $\mathcal{W}$ the set of all Borel probability measures on $\mathbb{R}^{n} \times \mathbb{T}^{n}$ that are EulerLagrangian flow invariant. For fixed $p \in \mathbb{R}^{n}, \mu \in \mathcal{W}$ is called a "Mather measure" if

$$
\int_{\mathbb{R}^{n} \times \mathbb{T}^{n}}(L(q, x)-p \cdot q) d \mu=\min _{\nu \in \mathcal{W}} \int_{\mathbb{R}^{n} \times \mathbb{T}^{n}}(L(q, x)-p \cdot q) d \nu .
$$

Denote by $\mathcal{W}_{p}$ the set of all such Mather measures. The value of the minimum action on the right-hand side turns out to be $-\bar{H}(p)$, i.e.,

$$
\min _{v \in \mathcal{W}} \int_{\mathbb{R}^{n} \times \mathbb{T}^{n}}(L(q, x)-p \cdot q) d v=-\bar{H}(p) .
$$

In dynamical systems literature, the effective Hamiltonian $\bar{H}$ is called the " $\alpha$-function" and is often denoted $\alpha(c)$, where $c$ is the same as $p$. 
The Mather set is defined to be the closure of the union of the support of all Mather measures, i.e.,

$$
\tilde{\mathcal{M}}_{p}=\overline{\bigcup_{\mu \in W_{p}} \operatorname{supp}(\mu)}
$$

The projected Mather set $\mathcal{M}_{p}$ is the projection of $\tilde{\mathcal{M}}_{p}$ to the torus. A curve $\xi: \mathbb{R} \rightarrow \mathbb{T}^{n}$ is called an orbit on $\mathcal{M}_{p}$ if it satisfies (2.2) and

$$
(\dot{\xi}(0), \xi(0)) \in \tilde{\mathcal{M}}_{p}
$$

If we lift $\tilde{\mathcal{M}}_{p}$ to $\mathbb{R}^{n} \times \mathbb{R}^{n}$ (or project $\tilde{\mathcal{A}}_{p}$ and $\tilde{\mathcal{N}}_{p}$ in our definition to $\mathbb{R}^{n} \times \mathbb{T}^{n}$ ), the following relation holds:

$$
\tilde{\mathcal{M}}_{p} \subset \tilde{\mathcal{A}}_{p} \subset \tilde{\mathcal{N}}_{p}
$$

In particular, the graph property (2.4) also holds for $\tilde{\mathcal{M}}_{p}$. Also, all trajectories on $\mathcal{M}_{p}$ are universal global characteristics and, hence, absolutely minimizing curves with respect to $\bar{H}(p)$. Moreover, all viscosity solutions to the cell problem (1.3) are $C^{1,1}$ on $\mathcal{M}_{p}$. See [8] for instance.

One hope is that Mather sets might have some sort of "integrable structure" in the sense that long term behaviors of trajectories there can be better understood. However, when $n \geq 3$, very little has been known in this direction except in certain special cases like the classical Hedlund example and its generalizations ([10,11]). We also would like mention that for generic $V$, [3, Cor. 1.2] says that there are at most $n+1$ ergodic Mather measures for every $p$, which is proved under some framework of convex analysis.

The following lemma is a well-known fact in the theory of Hamilton-Jacobi equations ([12]).

Lemma 2.1. Let $U$ be an open subset of $\mathbb{R}^{n}$. Assume that for some $c \in \mathbb{R}, w \in W^{1, \infty}(U)$ satisfies

$$
H(D w, x) \leq c \quad \text { for a.e } x \in U .
$$

Then for any $\eta \in \mathrm{AC}\left(\left[t_{1}, t_{2}\right], U\right)$,

$$
\int_{t_{1}}^{t_{2}} L(\dot{\eta}(t), \eta(t))+c d t \geq w\left(\eta\left(t_{2}\right)\right)-w\left(\eta\left(t_{1}\right)\right) .
$$

The equality holds if and only if $\eta$ is a characteristic of $w$, i.e., $w$ is differentiable along $\eta, H(D w(\eta(t)), \eta(t))=c$ and $D w(\eta(t))=D_{q} L(\dot{\eta}(t), \eta(t))$ for $t \in\left[t_{1}, t_{2}\right]$.

Using solutions to the cell problem (1.3), we have an immediate corollary:

Corollary 2.1. If $\gamma:[0, T] \rightarrow \mathbb{R}$ satisfies

$$
\gamma(T)-\gamma(0)=\vec{l} \in \mathbb{Z}^{n},
$$

then

$$
\int_{0}^{T} L(\dot{\gamma}, \gamma)+\bar{H}(p) d t \geq p \cdot \vec{l} .
$$

The equality holds if and only if $\gamma$ is a periodic orbit in $\mathcal{M}_{p}$. 


\subsection{Classical Aubry-Mather theory when $n=2$}

In this section we assume that $n=2$ and focus on

$$
H(p, x)=\frac{1}{2}|p|^{2}+V .
$$

Throughout this section we fix

$$
c>\max _{\mathbb{R}^{n}} V
$$

Due to (2.5), the two-dimensional topology and fact that different trajectories on $\mathcal{M}_{p}$ cannot intersect, it can be proved that the level curve ([4])

$$
S_{c}=\{\bar{H}(p)=c\}
$$

is $C^{1}$. This is equivalent to saying that for every $p \in S_{c}$, there exists a unit vector $q_{p}$ such that

$$
\partial \bar{H}(p)=\left\{\lambda q_{p} \mid \lambda \in[a, b]\right\} .
$$

Here $a \leq b$ are two positive constants depending on $p$ and $q_{p}$ is the outward unit normal vector of $S_{c}$ at $p$. Note that if $a=b$, then $\bar{H}$ is differentiable at $p$.

- Property 8: Cornerstone of Aubry-Mather theory. If $q_{p}$ is a rational vector, then all orbits on $\mathcal{M}_{p}$ are periodic orbits with the same first homology class $(m, n) \in \mathbb{Z}^{2}$ that is irreducible.

Remark 2.1. Choose $p_{k} \rightarrow p$ as $k \rightarrow+\infty$ such that $\bar{H}\left(p_{k}\right)>\bar{H}(p)$ and $q_{p_{k}}=q_{p}$; then by standard convex analysis,

$$
\lim _{k \rightarrow+\infty} \partial \bar{H}\left(p_{k}\right)=b q_{p}
$$

Together with the stability of periodic orbits (see the analysis in the appendix), we can deduce that there is a periodic orbit on $\mathcal{M}_{p}$ whose rotation vector is $b q_{p}$. Similarly, there is a periodic orbit on $\mathcal{M}_{p}$ whose rotation vector is $a q_{p}$.

- Property 9: Identification with circle homeomorphism. Choose $\hat{p} \in S_{c}$ such that $q_{\hat{p}}=$ $(0,1)$. Let $\xi$ be a periodic orbit on $\mathcal{M}_{\hat{p}}$ and lift it to $\mathbb{R}^{2}$. Now for each $k \in \mathbb{Z}$, denote

$$
\xi_{k}=\xi+(k, 0)
$$

For $p \in \mathbb{R}^{2}$ with $q_{p} \neq(0,1)$ or $(0,-1)$, let $\gamma: \mathbb{R} \rightarrow \mathbb{R}^{2}$ be a global characteristic associated with a solution to the cell problem (1.3). Owing to Property 1 , for each $k \in \mathbb{Z}, \gamma$ intersects with $\xi_{k}$ exactly once. Let $a_{k} \in \mathbb{R}$ be such that (see Figure 2)

$$
\gamma \cap \xi_{k}=\xi_{k}\left(a_{k} T\right)
$$




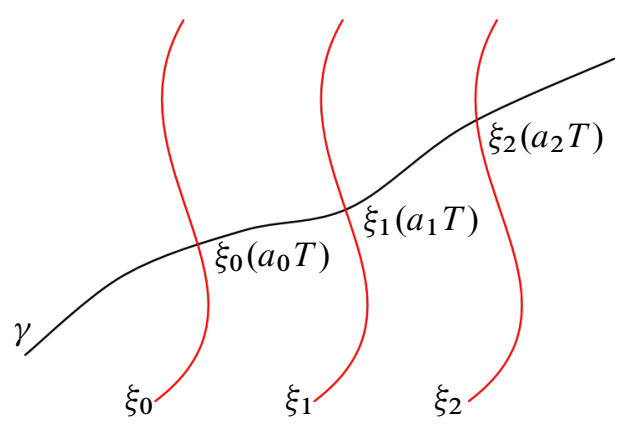

Figure 2

Since $\gamma$ cannot intersect itself when it is projected to $\mathbb{T}^{2}$, either $a_{k}=0$ for all $k \in \mathbb{Z}$ or $\left\{a_{k}\right\}_{k \in \mathbb{Z}}$ is a strictly monotonic sequence. If $\left\{a_{k}\right\}_{k \in \mathbb{Z}}$ is strictly increasing, there exists a circle homeomorphism $f$ such that

$$
f\left(a_{k}\right)=a_{k+1} \quad \text { for all } k \in \mathbb{Z}
$$

See [1, Thm. 3.15] for further details on the definition of $f$. If $\left\{a_{k}\right\}_{k \in \mathbb{Z}}$ is strictly decreasing, then consider $f\left(a_{k}\right)=a_{k-1}$. We would like to mention that this identification plays a crucial role in obtaining the optimal convergence rate $\left|u^{\varepsilon}-u\right|=O(\varepsilon)$ for homogeneous $H(p, x)([15])$.

We say that $p \in S_{c}$ is a linear point if there exists $p^{\prime} \in \mathbb{R}^{2}$ such that $p^{\prime} \neq p$ and the line segment (edge)

$$
\left[p, p^{\prime}\right] \subset S_{c} .
$$

Clearly, $q_{p}=q_{p^{\prime}}$ and $q_{p} \cdot\left(p-p^{\prime}\right)=0$. Combining with the definition of Mather sets, we can deduce that, for every $p^{\prime \prime} \in\left[p, p^{\prime}\right]$,

$$
\tilde{\mathcal{M}}_{p^{\prime \prime}}=\tilde{\mathcal{M}}_{p}
$$

The following two results were proved in [2].

- Property 10. A point $p \in S_{c}$ is a linear point if and only if $\mathcal{M}_{p} \neq \mathbb{T}^{2}$. Moreover, if $p$ is a linear point, then $q_{p}$ is a rational vector. The converse might not be true in general. Nevertheless, for generic $V$, [3, Cor. 1.2] and the above Property 8 imply that if $q_{p}$ is an rational vector, then $\mathcal{M}_{p}$ has at most 3 periodic orbits and hence $p$ must be a linear point. See [5] for hyperbolicity of periodic orbits.

- Property 11. The set $S_{c}$ is not strictly convex (i.e., the set of linear points on $S_{c}$ is not empty) unless $V$ is a constant.

The results in [2] are presented under the framework of a minimizing geodesic associated with a periodic Riemannian metric on $\mathbb{R}^{2}$. Note that for mechanical Hamiltonians, 
every absolutely minimizing curve associated with $L(q, x)+c$ is a minimizing geodesic associated with the Riemannian metric

$$
g=\sqrt{2(c-V(x))}\left(d x_{1} \otimes d x_{1}+d x_{2} \otimes d x_{2}\right) .
$$

The converse is also true after proper reparametrization.

\section{Proof of Theorem 1.1}

Fix $p_{0} \in \mathbb{R}^{2}$ with $\bar{H}\left(p_{0}\right)>\max _{\mathbb{T}^{2}} V$. Denote

$$
c_{0}=\bar{H}\left(p_{0}\right)
$$

and

$$
S_{c_{0}}=\left\{p \in \mathbb{R}^{2} \mid \bar{H}(p)=c_{0}\right\} .
$$

Assume that the outward unit normal vector $q_{p_{0}}($ see $(2.7))$ at $p_{0}$ is a rational vector. Denote

$$
q_{p_{0}}=\frac{(m, n)}{\sqrt{m^{2}+n^{2}}}
$$

Here

$$
(m, n) \text { is the first homology class of periodic orbits on } \mathcal{M}_{p_{0}} \text {. }
$$

Throughout this section, we lift $\mathcal{M}_{p_{0}}$ to $\mathbb{R}^{2}$ and still denote it as $\mathcal{M}_{p_{0}}$. In addition, for convenience, the lift of a periodic orbit on $\mathcal{M}_{p_{0}}$ is also called a periodic orbit.

Suppose that $v$ is a viscosity solution to

$$
\frac{1}{2}\left|p_{0}+D v\right|^{2}+V=c_{0}
$$

For $A, B \subset \mathbb{R}^{2}$ and

$$
u=p_{0} \cdot x+v,
$$

we define the barrier between two sets as

$$
d_{u}(A, B)=\inf _{x \in A, y \in B}(h(x, y)-(u(y)-u(x))) .
$$

Here,

$$
h(x, y)=\inf _{\substack{t>0, \gamma \in \mathrm{AC}([0, t]) \\ \gamma(0)=x, \gamma(t)=y}}\left(\int_{0}^{t} \frac{1}{2}|\dot{\gamma}|^{2}-V(\gamma)+c_{0} d s\right)
$$

and $\mathrm{AC}([0, t])$ is the set of all absolutely continuous curves $[0, t] \rightarrow \mathbb{R}^{2}$. Owing to Lemma 2.1,

$$
d_{u}(A, B) \geq 0
$$

If $L_{1}: \mathbb{R} \rightarrow \mathbb{R}^{2}$ and $L_{2}: \mathbb{R} \rightarrow \mathbb{R}^{2}$ are two curves, $d_{u}\left(L_{1}, L_{2}\right)$ is to be understood as $d_{u}\left(A_{1}, A_{2}\right)$ for $A_{1}=L_{1}(\mathbb{R})$ and $A_{2}=L_{2}(\mathbb{R})$. 
Lemma 3.1. Suppose that $\gamma_{1}$ and $\gamma_{2}$ are two periodic orbits on $\mathcal{M}_{p_{0}}$. Then

$$
d_{u}\left(\gamma_{1}, \gamma_{2}\right)=\lim _{\substack{t \rightarrow+\infty \\ s \rightarrow-\infty}}\left(h\left(\gamma_{1}(s), \gamma_{2}(t)\right)-\left(u\left(\gamma_{2}(t)\right)-u\left(\gamma_{1}(s)\right)\right)\right)
$$

Proof. Denote

$$
G(t, s)=h\left(\gamma_{1}(s), \gamma_{2}(t)\right)-\left(u\left(\gamma_{2}(t)\right)-u\left(\gamma_{1}(s)\right)\right)
$$

We claim that $G$ is decreasing in $t$ and increasing in $s$. In fact, for $t_{1}<t_{2}$, due to the triangle inequality,

$$
\begin{aligned}
h\left(\gamma_{1}(s), \gamma_{2}\left(t_{2}\right)\right) & \leq h\left(\gamma_{1}(s), \gamma_{2}\left(t_{1}\right)\right)+h\left(\gamma_{2}\left(t_{1}\right), \gamma_{2}\left(t_{2}\right)\right) \\
& =h\left(\gamma_{1}(s), \gamma_{2}\left(t_{1}\right)\right)+\left(u\left(\gamma_{2}\left(t_{2}\right)\right)-u\left(\gamma_{2}\left(t_{1}\right)\right)\right) .
\end{aligned}
$$

Hence $G\left(s, t_{2}\right) \leq G\left(s, t_{1}\right)$. Similarly, we can show that $G$ is increasing in $s$. Then our lemma follows immediately.

Let $D$ be an open set bounded by two unbounded simple curves $L_{1}$ and $L_{2}$ on $\mathbb{R}^{2}$. For any $\delta$ with $0 \leq \delta \leq d_{u}\left(L_{1}, L_{2}\right)$, let (see Figure 3)

$$
\begin{cases}g(x)=u(x) & \text { for } x \in L_{1}, \\ g(x)=u(x)+\delta & \text { for } x \in L_{2}\end{cases}
$$

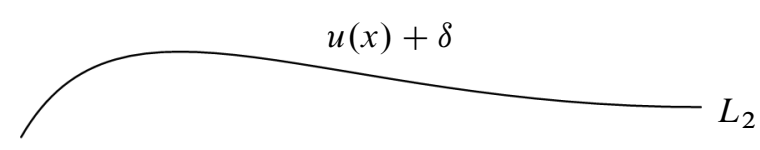

$D$

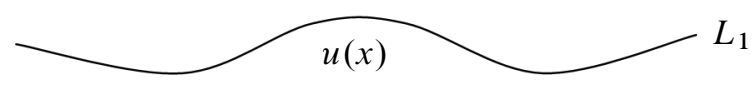

Figure 3

Define

$$
u_{\delta}(x)=\inf _{y \in L_{1} \cup L_{2}}\{g(y)+h(y, x)\} \quad \text { for } x \in \bar{D} .
$$

The following lemma is due to the well-known compatibility condition for existence of solutions to the Hamilton-Jacobi equations ([12]).

Lemma 3.2. The above function $u_{\delta}$ is a Lipschitz continuous viscosity solution to

$$
\left\{\begin{array}{l}
\frac{1}{2}\left|D u_{\delta}\right|^{2}+V(x)=c_{0} \\
u_{\delta}=u \text { on } L_{1} \\
u_{\delta}=u+\delta \text { on } L_{2} .
\end{array}\right.
$$


We have the following corollary:

Corollary 3.1. Let $\xi_{1}$ and $\xi_{2}$ be two periodic orbits on $\mathcal{M}_{p_{0}}$. If the region $D \subset \mathbb{R}^{2}$ bounded by $\xi_{1}$ and $\xi_{2}$ is foliated by periodic orbits on $\mathcal{M}_{p_{0}}$, then (see Figure 4)

$$
d_{u}\left(\xi_{1}, \xi_{2}\right)=0
$$

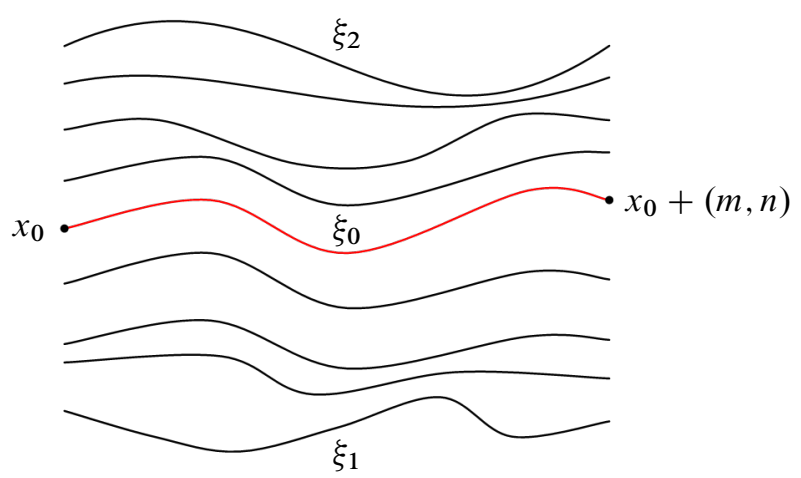

Figure 4

Proof. Let $(m, n)$ be the homology class of periodic orbits on $\mathcal{M}_{p}$. Choose $\delta=d_{u}\left(\xi_{1}, \xi_{2}\right)$. Apparently, $D+(m, n)=D$. Also,

$$
\begin{aligned}
u_{\delta}(x+(m, n)) & =\inf _{y \in L_{1} \cup L_{2}}\{g(y)+h(y, x+(m, n))\} \\
& =\inf _{y \in L_{1} \cup L_{2}}\{g(y+(m, n))+h(y+(m, n), x+(m, n))\} \\
& =\inf _{y \in L_{1} \cup L_{2}}\{g(y+(m, n))+h(y, x)\} \\
& =\inf _{y \in L_{1} \cup L_{2}}\{g(y)+h(y, x)\}+p_{0} \cdot(m, n) \\
& =u_{\delta}(x)+p_{0} \cdot(m, n) .
\end{aligned}
$$

The second equality in the above is due to $\left(L_{1} \cup L_{2}\right)+(m, n)=L_{1} \cup L_{2}$. The third one is from the periodicity of $V$. The last equality is because $g(y+h)-g(y)=u(y+h)-$ $u(y)=p_{0} \cdot h$ for all $h \in \mathbb{R}^{2}$.

To prove $d_{u}\left(\xi_{1}, \xi_{2}\right)=0$, it suffices to show that

$$
u_{\delta} \equiv u \quad \text { in } \bar{D} \text {. }
$$

In fact, choose an arbitrary point $x_{0} \in D$ and let $\xi_{0}$ be the periodic orbit that passes through $x_{0}$ with minimum period $T_{0}$. Then

$$
\begin{aligned}
u_{\delta}\left(x_{0}+(m, n)\right)-u_{\delta}\left(x_{0}\right)=(m, n) \cdot p_{0} & =u\left(x_{0}+(m, n)\right)-u\left(x_{0}\right) \\
& =\int_{0}^{T_{0}} \frac{1}{2}\left|\dot{\xi}_{0}\right|^{2}-V\left(\xi_{0}\right)+c_{0} d s .
\end{aligned}
$$


The second " $=$ " is due to the fact that every trajectory on $\mathcal{M}_{p_{0}}$ is a universal global characteristic. Hence $\xi_{0}$ is also a characteristic of $u_{\delta}$ and $D u_{\delta}\left(x_{0}\right)=D u\left(x_{0}\right)=\dot{\xi}_{0}(0)$. Accordingly,

$$
D u_{\delta} \equiv D u \quad \text { in } D
$$

Therefore $u_{\delta}=u$ in $\bar{D}$.

Lemma 3.3. Suppose that $\xi$ is a periodic orbit on $\mathcal{M}_{p_{0}}$. Let $\xi_{2}=\xi_{1}+(-n, m)$.

(1) There exists $\tau>0$ such that

$$
E_{p_{0}, \tau,+}=\left\{p_{0}+t(-n, m) \mid t \in[0, \tau]\right\} \subset S_{c_{0}}
$$

if and only if

$$
d_{u}\left(\xi_{1}, \xi_{2}\right)>0
$$

(2) There exists $\tau>0$ such that

$$
E_{p_{0}, \tau,-}=\left\{p_{0}+t(n,-m) \mid t \in[0, \tau]\right\} \subset S_{c_{0}}
$$

if and only if

$$
d_{u}\left(\xi_{2}, \xi_{1}\right)>0
$$

Proof. It is enough to prove (1). The proof for (2) is similar.

" $\Rightarrow$ " Denote

$$
p_{\tau}=p_{0}+\tau(-n, m)
$$

Let $v_{\tau}$ be a viscosity solution to

$$
\frac{1}{2}\left|p_{\tau}+D v_{\tau}\right|^{2}+V(x)=c_{0} .
$$

Recall that $c_{0}=\bar{H}\left(p_{0}\right)$. Owing to (2.8), $\xi_{1}$ and $\xi_{2}$ are also periodic orbits on $\mathcal{M}_{p_{\tau}}$. Hence

$$
D u_{\tau}=D u \quad \text { on } \xi_{1} \cup \xi_{2}
$$

Without loss of generality, we may assume that $u_{\tau}=u$ on $\xi_{1}$. Then $u_{\tau}=u+\tau\left(m^{2}+n^{2}\right)$ on $\xi_{2}=\xi_{1}+(-n, m)$. Accordingly,

$$
d_{u}\left(\xi_{1}, \xi_{2}\right)=d_{u_{\tau}}\left(\xi_{1}, \xi_{2}\right)+\tau\left(m^{2}+n^{2}\right) \geq \tau\left(m^{2}+n^{2}\right)>0 .
$$

“ $\Leftarrow$ ” This part is not really needed for our main result. Since it is the essential PDE part in proving the existence of edges of $S_{c_{0}}$, we present it here. Choose

$$
\tau=\frac{d_{u}\left(\xi_{1}, \xi_{2}\right)}{m^{2}+n^{2}}
$$

For $p^{\prime} \in E_{p_{0}, \tau,+}$, define

$$
F= \begin{cases}u & \text { on } \xi_{1}, \\ u+p^{\prime} \cdot(-n, m) & \text { on } \xi_{2}\end{cases}
$$


Clearly,

$$
\inf _{x, y \in \xi_{1} \cup \xi_{2}}(h(x, y)-(F(y)-F(x))) \geq 0 .
$$

Hence for $x \in D$,

$$
u_{p^{\prime}}(x)=\inf _{y \in \xi_{1} \cup \xi_{2}}\{F(y)+h(x, y)\}
$$

is a viscosity solution of

$$
\begin{cases}\frac{1}{2}\left|D u_{p^{\prime}}\right|^{2}+V(x)=c_{0} & \text { in } D, \\ u_{p^{\prime}}=F & \text { on } \partial D .\end{cases}
$$

As in Corollary 3.1, we have that for $x \in D$,

$$
u_{p^{\prime}}(x+(m, n))=u_{p^{\prime}}(x)+p_{0} \cdot(m, n)=u_{p^{\prime}}(x)+p^{\prime} \cdot(m, n) .
$$

Next we may extend $u_{p^{\prime}}$ to $\mathbb{R}^{2}$ by

$$
u_{p^{\prime}}(x+k(-n, m))=u_{p^{\prime}}(x)+k p^{\prime} \cdot(-n, m) \quad \text { for all } x \in D \text { and } k \in \mathbb{Z} .
$$

See Figure 5. Apparently, $v_{p^{\prime}}=u_{p^{\prime}}-p^{\prime} \cdot x$ is an $\left(m^{2}+n^{2}\right) \mathbb{Z}^{2}$ periodic viscosity solution to

$$
\frac{1}{2}\left|p^{\prime}+D v_{p^{\prime}}\right|^{2}+V=c_{0} \quad \text { in } \mathbb{R}^{2} /\left(\xi_{1}+(-n, m) \mathbb{Z}\right) .
$$

Since $\xi_{1}$ is a periodic characteristic of $v_{p^{\prime}}$, it is easy to see that $v_{p^{\prime}}$ is a $\left(m^{2}+n^{2}\right) \mathbb{Z}^{2}$ periodic viscosity solution to

$$
\frac{1}{2}\left|p^{\prime}+D v_{p^{\prime}}\right|^{2}+V=c_{0} \quad \text { on } \mathbb{R}^{2}
$$

By the inf-max formula (1.4), it is not hard to deduce that

$$
\bar{H}\left(p^{\prime}\right)=c_{0} .
$$

Lemma 3.4. Suppose that $L_{1}, L_{2}, L_{3}, \ldots, L_{m}$ are $m$ different periodic orbits on $\mathcal{M}_{p_{0}}$. For $i=2, \ldots, m-1, L_{i}$ lies between $L_{i-1}$ and $L_{i+1}$. Then

$$
d_{u}\left(L_{1}, L_{m}\right)=\sum_{k=1}^{m-1} d_{u}\left(L_{k}, L_{k+1}\right)
$$

Proof. By induction, it suffices to establish the above equality for $m=3$. By definition of $d_{u}$, it is clear that

$$
d_{u}\left(L_{1}, L_{3}\right) \geq d_{u}\left(L_{1}, L_{2}\right)+d_{u}\left(L_{2}, L_{3}\right) .
$$

Now let us prove the other direction. According to the definition, for any $\delta>0$, there exist two Lipschitz continuous curves $\gamma_{1}:[0, a] \rightarrow \mathbb{R}^{2}$ and $\gamma_{2}:[0, b] \rightarrow \mathbb{R}^{2}$ such that

$$
\begin{gathered}
\gamma_{1}(0) \in L_{1}, \quad \gamma_{1}(a), \gamma_{2}(0) \in L_{2}, \quad \gamma_{2}(b) \in L_{3}, \\
d_{u}\left(L_{1}, L_{2}\right) \geq \int_{0}^{a} \frac{1}{2}\left|\dot{\gamma}_{1}\right|^{2}-V\left(\gamma_{1}\right)+c_{0} d s-u\left(\gamma_{1}(0)\right)+u\left(\gamma_{1}(a)\right)-\delta, \\
d_{u}\left(L_{2}, L_{3}\right) \geq \int_{0}^{b} \frac{1}{2}\left|\dot{\gamma}_{2}\right|^{2}-V\left(\gamma_{2}\right)+c_{0} d s-u\left(\gamma_{2}(0)\right)+u\left(\gamma_{2}(b)\right)-\delta .
\end{gathered}
$$




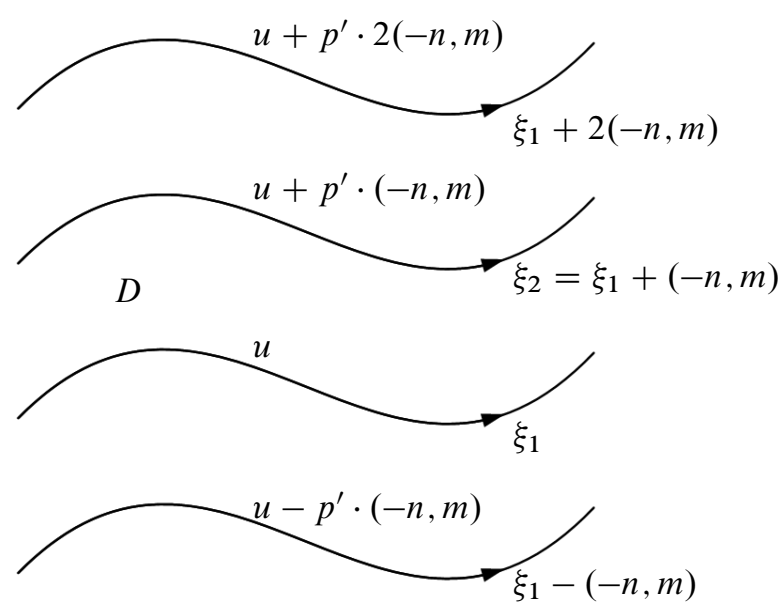

Figure 5

By periodic translation, we can make $\gamma_{1}(a)$ at an earlier time than $\gamma_{2}(0)$, i.e., there exists $t_{1}<t_{2}$ such that

$$
\gamma_{1}(a)=L_{2}\left(t_{1}\right) \quad \text { and } \quad \gamma_{2}(0)=L_{2}\left(t_{2}\right)
$$

By connecting $\gamma_{1}, L_{2}$ and $\gamma_{2}$, we define $\gamma:\left[0, a+t_{2}-t_{1}+b\right] \rightarrow \mathbb{R}^{2}$ as (see Figure 6)

$$
\gamma(t)= \begin{cases}\gamma_{1}(t) & \text { for } t \in[0, a], \\ L_{2}\left(t-a+t_{1}\right) & \text { for } t \in\left[a, a+t_{2}-t_{1}\right], \\ \gamma_{2}\left(t+t_{t}-t_{2}-a\right) & \text { for } t \in\left[a+t_{2}-t_{1}, a+t_{2}-t_{1}+b\right] .\end{cases}
$$

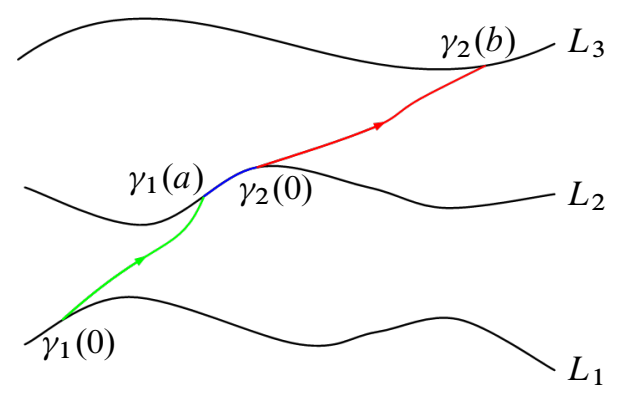

Figure 6

Since

$$
\int_{t_{1}}^{t_{2}} \frac{1}{2}\left|\dot{L}_{2}\right|^{2}-V\left(L_{2}\right)+c_{0} d s-u\left(L_{2}\left(t_{2}\right)\right)+u\left(L_{2}\left(t_{1}\right)\right)=0
$$


we have that for $\bar{t}=a+b+t_{2}-t_{1}$,

$$
\int_{0}^{\bar{t}} \frac{1}{2}|\dot{\gamma}|^{2}-V(\gamma)+c_{0} d s-u(\gamma(\bar{t}))+u(\gamma(0)) \leq d_{u}\left(L_{1}, L_{2}\right)+d_{u}\left(L_{2}, L_{3}\right)+2 \delta .
$$

Accordingly,

$$
d_{u}\left(L_{1}, L_{3}\right) \leq d_{u}\left(L_{1}, L_{2}\right)+d_{u}\left(L_{2}, L_{3}\right)+2 \delta .
$$

Sending $\delta \rightarrow 0$ leads to

$$
d_{u}\left(L_{1}, L_{3}\right) \leq d_{u}\left(L_{1}, L_{2}\right)+d_{u}\left(L_{2}, L_{3}\right)
$$

Hence our lemma holds.

Next we prove that $\bar{H}$ is one-dimensional near any point that is in the interior of an edge. This conclusion holds for any $V \in C^{k}\left(\mathbb{T}^{2}\right)$ when $k \geq 2$.

Lemma 3.5. Suppose there exist $p_{1} \neq p_{2} \in S_{c_{0}}$ such that

$$
p_{0} \in\left\{t p_{1}+(1-t) p_{2} \mid t \in(0,1)\right\} \subset S_{c_{0}},
$$

i.e., $p_{0}$ is in the interior of an edge. Then there exist $r>0$ and a convex function $f: \mathbb{R} \rightarrow$ $\mathbb{R}^{2}$ such that

$$
\bar{H}(p)=f\left(q_{p_{0}} \cdot p\right) \quad \text { for } p \in B_{r}\left(p_{0}\right) .
$$

Proof. It suffices to show that there exists $r>0$ such that

$$
q_{p}=q_{p_{0}} \quad \text { for } p \in B_{r}\left(p_{0}\right)
$$

Recall the definition of $q_{p}$ in (2.7). If this holds, $\bar{H}$ is constant along the direction that is perpendicular to $q_{p_{0}}$ in $B_{r}\left(p_{0}\right)$. Then

$$
f(t)=\bar{H}\left(p_{0}+q_{p_{0}}\left(t-p_{0} \cdot q_{p_{0}}\right)\right) .
$$

We argue by contradiction. If not true, then there exist $\left\{p_{k}\right\}_{k \geq 1}$ such that $p_{k} \rightarrow p_{0}$ as $k \rightarrow+\infty$ and

$$
q_{p_{k}} \cdot(-n, m) \neq 0 .
$$

Without loss of generality, let us assume that

$$
q_{p_{k}} \cdot(-n, m)>0 .
$$

Let $v_{k}$ be a periodic viscosity solution to

$$
\frac{1}{2}\left|p_{k}+D v_{k}\right|^{2}+V(x)=\bar{H}\left(p_{k}\right)
$$

subject to $\int_{\mathbb{T}^{2}} v_{k} d x=0$. Up to a subsequence if necessary, we assume that

$$
\lim _{k \rightarrow+\infty} v_{k}=\hat{v} \quad \text { uniformly on } \mathbb{R}^{2} .
$$


Then $\hat{v}$ is a $\mathbb{Z}^{2}$-periodic viscosity solution of

$$
\frac{1}{2}\left|p_{0}+D \hat{v}\right|^{2}+V(x)=c_{0} \quad \text { on } \mathbb{R}^{2}
$$

subject to $\int_{\mathbb{T}^{2}} \hat{v} d x=0$.

For $k \geq 1$, since the level curve $\left\{\bar{H}=\bar{H}\left(p_{k}\right)\right\}$ is $C^{1}$, we can choose $\tilde{p}_{k}$ such that

$$
\bar{H}\left(p_{k}\right)=\bar{H}\left(\tilde{p}_{k}\right)
$$

and the outward unit normal vector at $\tilde{p}_{k}$,

$$
q_{\tilde{p}_{k}}=\frac{(-n, m)}{\sqrt{m^{2}+n^{2}}} .
$$

Let $\eta_{k}: \mathbb{R} \rightarrow \mathbb{R}^{2}$ be a periodic orbit on $\mathcal{M}_{\tilde{p}_{k}}$ (lift to $\mathbb{R}^{2}$ ). Up to a subsequence, we may assume that

$$
\lim _{k \rightarrow+\infty} \tilde{p}_{k}=\tilde{p}
$$

and

$$
\lim _{k \rightarrow+\infty} \eta_{k}=\eta \quad \text { locally uniformly on } \mathbb{R} .
$$

Then $\bar{H}(\tilde{p})=\bar{H}\left(p_{0}\right)$ and $\eta$ is a periodic orbit on $\mathcal{M}_{\tilde{p}}$ with first homology class $(-n, m)$.

Suppose that $\xi_{1}$ and $\xi_{2}$ are two periodic orbits on $\mathcal{M}_{p_{0}}$ such that there is no other periodic orbit in the region bounded by $\xi_{1}$ and $\xi_{2}$ (a gap).

Claim: For $\hat{u}=p_{0} \cdot x+\hat{v}$,

$$
d_{\hat{u}}\left(\xi_{1}, \xi_{2}\right)=0 .
$$

The orbit $\xi_{1}$ divides the plane into two regions. Without loss of generality, we may assume that $\xi_{2}$ is in the same region as $\xi_{1}+(-n, m)$. For $k \geq 1$, let $\gamma_{k}: \mathbb{R} \rightarrow \mathbb{R}^{2}$ be an orbit on $\mathcal{M}_{p_{k}}$ (lift to $\mathbb{R}^{2}$ ) with $\gamma_{k}(0)=x_{k}$ for some $x_{k} \in[0,1]^{2}$ satisfying that the distance from $x_{k}$ to $\xi_{2}$ is half the distance between $\xi_{1}$ and $\xi_{2}$ :

$$
\operatorname{distance}\left(x_{k}, \xi_{2}\right)=\frac{1}{2} \operatorname{distance}\left(\xi_{1}, \xi_{2}\right) \text {. }
$$

Up to a subsequence if necessary, we assume that

$$
\lim _{k \rightarrow+\infty} \gamma_{k}=\gamma \quad \text { locally uniformly on } \mathbb{R}
$$

and

$$
\lim _{k \rightarrow+\infty} x_{k}=x_{\infty} \in[0,1]^{2} .
$$

See Figure 7. Due to the stability, $\gamma: \mathbb{R} \rightarrow \mathbb{R}^{2}$ is a global characteristic of $\hat{v}$ with $\gamma(0)=$ $x_{\infty}$. Owing to (3.2), intersections of $\gamma$ with respect to $\eta+(m, n) \mathbb{Z}$ are non-decreasing (see Property 9 in Section 2.3 for the precise meaning). Since there is no periodic orbit between $\xi_{1}$ and $\xi_{2}$, there exist $\left\{t_{i}^{+}\right\}_{i \geq 1}$ and $\left\{t_{i}^{-}\right\}_{i \geq 1}$ such that

$$
\lim _{i \rightarrow+\infty} t_{i}^{+}=+\infty \quad \text { and } \quad \lim _{i \rightarrow+\infty}\left|\gamma\left(t_{i}^{+}\right)-\xi_{2}\left(t_{i}^{+}\right)\right|=0
$$

and

$$
\lim _{i \rightarrow+\infty} t_{i}^{-}=-\infty \quad \text { and } \lim _{i \rightarrow+\infty}\left|\gamma\left(t_{i}^{-}\right)-\xi_{1}\left(t_{i}^{-}\right)\right|=0
$$




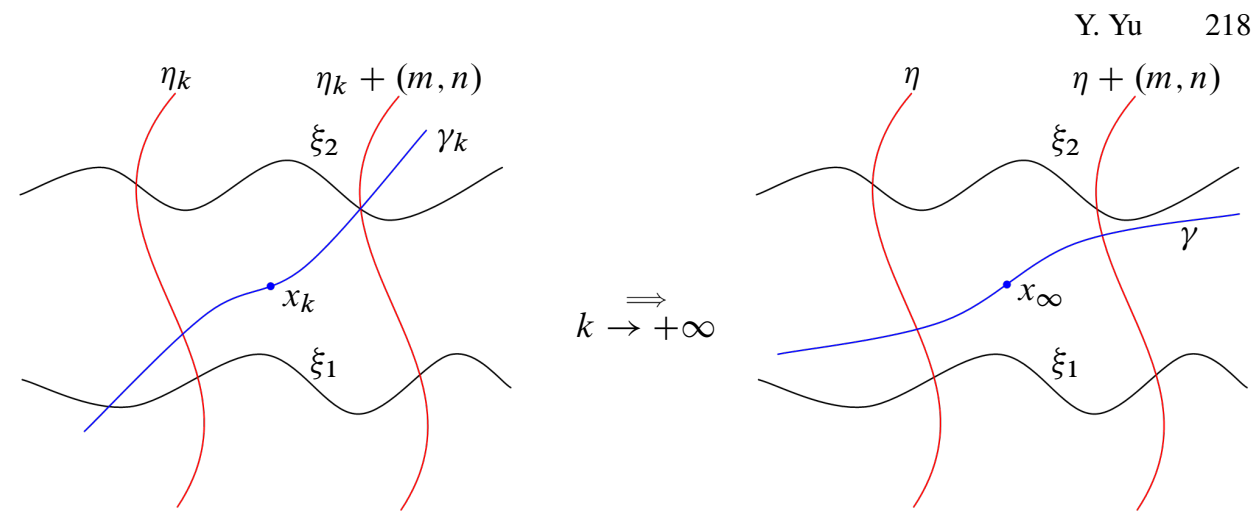

Figure 7

Accordingly, we can derive that

$$
d_{\hat{u}}\left(\xi_{1}, \xi_{2}\right)=0 .
$$

Combining Corollary 3.1 and Lemma 3.4, we deduce that

$$
d_{\hat{u}}\left(\xi_{1}, \xi_{1}+(-n, m)\right)=0
$$

This contradicts Lemma 3.3 and the assumption that $p_{0}$ is in the interior of an edge. Hence (3.1) and the lemma hold.

Proof of Theorem 1.1. Let

$$
\mathscr{E}=\bigcap_{q \in \mathbb{Q}^{2}, r \in \mathbb{Q}} C(q, r)
$$

Here $\mathbb{Q}^{2}$ is the collection of points on $\mathbb{R}^{2}$ with both coordinates rational numbers. See the appendix for the definition of $C(q, r)$. Fix $V \in \mathcal{E}$. Then for any $p \in \mathbb{R}^{2}$, if $\bar{H}(p)>$ $\max _{\mathbb{R}^{2}} V$ and $\partial \bar{H}(p) \cap \mathbb{Q}^{2} \neq \emptyset, p$ must be a linear point. Now write

$$
O_{V}=\left\{p \in \mathbb{R}^{2} \mid \bar{H}(p)>\min _{\mathbb{R}^{2}} V \text { and } p \text { is an interior linear point }\right\} \cup F_{0}^{\circ} .
$$

Recall that the minimum level set $F_{0}=\left\{p \in \mathbb{R}^{2} \mid \bar{H}(p)=\max _{\mathbb{R}^{2}} V\right\}$. Owing to Lemma 3.5, $O_{V}$ is an open set. We only need to show that $O_{V}$ is dense. Let us argue by contradiction. If not, then there exist $\bar{p} \in \mathbb{R}^{2}$ and $B_{r}(\bar{p})$ for some $r>0$ such that $\bar{H}$ is strictly convex in $B_{r}(\bar{p})$. Then the set

$$
W=\bigcup_{p \in B_{r}(\bar{p})} \partial \bar{H}(p)
$$

is a non-empty open set. In particular, $W \cap \mathbb{Q}^{2}$ is not empty. This contradicts the choice of $V$ and $O_{V}$. 


\section{A. Construction of the residual set}

Definition A.1. Assume we are given $r>0$ and a non-zero rational vector $q$. Denote by

$$
C(q, r)
$$

the collection of all $V \in C^{k}\left(\mathbb{T}^{2}\right)$ such that for any $p \in \mathbb{R}^{2}$, if

$$
\bar{H}_{V}(p) \geq \max _{\mathbb{T}^{2}} V+r \quad \text { and } \quad q \in \partial \bar{H}(p)
$$

then

$$
\mathcal{M}_{p} \neq \mathbb{T}^{2} \quad \text { (equivalently, } p \text { is a linear point). }
$$

Theorem A.1. For $r>0$ and any non-zero rational vector $q$,

$$
C(q, r)
$$

is an open dense set.

Proof. It is equivalent to showing that the complement

$$
S=C^{k}\left(\mathbb{T}^{2}\right) \backslash C(q, r)
$$

is closed and nowhere dense. Write

$$
q=\lambda(m, n),
$$

where $\lambda>0$ and $(m, n) \in \mathbb{Z}^{2}$ is irreducible.

Step 1. We first prove that $S$ is closed. Suppose that $\left\{V_{j}\right\}_{j \geq 1}$ is a sequence of functions in $S$ and

$$
\lim _{j \rightarrow+\infty} V_{j}=V \quad \text { in } C^{k}\left(\mathbb{T}^{2}\right)
$$

Then

$$
\lim _{j \rightarrow+\infty} \bar{H}_{V_{j}}(p)=\bar{H}_{V}(p) \quad \text { locally uniformly in } \mathbb{R}^{2} .
$$

Since $V_{j} \in S$, for each $j \in \mathbb{N}$, there exists $p_{j} \in \mathbb{R}^{2}$ such that

$$
\bar{H}_{V_{j}}\left(p_{j}\right) \geq \max _{\mathbb{T}^{2}} V+r, \quad q \in \partial \bar{H}_{V_{j}}\left(p_{j}\right)
$$

and

$$
\mathcal{M}_{p_{j}, V_{j}}=\mathbb{T}^{2} .
$$

Due to the convexity, $\bar{H}_{V_{j}}(0) \geq \bar{H}_{V_{j}}\left(p_{j}\right)+q \cdot\left(-p_{j}\right)$. Together with the quadratic growth of $\bar{H}_{V_{j}}$, it is easy to see that $\left\{p_{j}\right\}_{j \geq 1}$ is uniformly bounded. Upon a subsequence if necessary, we assume that

$$
\lim _{j \rightarrow+\infty} p_{j}=\tilde{p} .
$$


Then

$$
\bar{H}_{V}(\tilde{p}) \geq \max _{\mathbb{T}^{2}} V+r
$$

and by upper semicontinuity of subdifferentials,

$$
q \in \partial \bar{H}_{V}(\tilde{p}) .
$$

Now we just need to show that

$$
\mathcal{M}_{\tilde{p}, V}=\mathbb{T}^{2} .
$$

This follows easily from the stability of periodic orbits. For the readers' convenience, we present details here. Fix $x_{0} \in \mathbb{T}^{2}$.

For each $j \geq 1$, let $\xi_{j}:\left[0, T_{j}\right] \rightarrow \mathbb{T}^{2}$ be a periodic orbit on $\mathcal{M}_{p_{j}, V_{j}}$ with $\xi_{j}(0)=x_{0}$ and $T_{j}$ is the minimum period. Then

$$
\xi_{j}\left(T_{j}\right)=x_{0}+(m, n), \quad \frac{\xi\left(T_{j}\right)-x_{0}}{T_{j}} \in \partial \bar{H}_{V_{j}}\left(p_{j}\right)
$$

and

$$
p_{j} \cdot(m, n)=\int_{0}^{T_{j}} \frac{1}{2}\left|\dot{\xi}_{j}\right|^{2}-V_{j}\left(\xi_{j}\right)+\bar{H}_{V_{j}}\left(p_{j}\right) d t .
$$

It is easy to see that $T_{j}$ and $\left\|\xi_{j}\right\|_{C^{2}\left(\left[0, T_{j}\right]\right)}$ are uniformly bounded. Up to a subsequence if necessary, we may assume that

$$
\lim _{j \rightarrow+\infty} T_{j}=T \quad \text { and } \quad \lim _{j \rightarrow+\infty} \xi_{j}=\xi \quad \text { uniformly in } C^{1}(\mathbb{R}) .
$$

Then

$$
\xi(0)=x_{0} \quad \text { and } \quad \xi(T)=x_{0}+(m, n)
$$

and

$$
\tilde{p} \cdot(m, n)=\int_{0}^{T} \frac{1}{2}|\dot{\xi}|^{2}-V(\xi)+\bar{H}_{V}(\tilde{p}) d t .
$$

So $\xi$ is a periodic orbit on $\mathcal{M}_{\tilde{p}, V}$ and $x_{0} \in \mathcal{M}_{\tilde{p}, V}$. Hence $\mathcal{M}_{\tilde{p}, V}=\mathbb{T}^{2}$.

Step 2. Next we prove that $C(q, r)$ is dense. Assume we are given $V_{0} \in S$. Then there exists $p_{0} \in \mathbb{R}^{2}$ such that $\bar{H}_{V_{0}}\left(p_{0}\right) \geq \max _{\mathbb{T}^{2}} V_{0}+r, q \in \partial \bar{H}\left(p_{0}\right)$ and $\mathcal{M}_{p_{0}, V_{0}}=\mathbb{T}^{2}$. Assume that

$$
\partial \bar{H}_{V_{0}}\left(p_{0}\right)=[\alpha q, \beta q]
$$

for $0<\alpha \leq 1 \leq \beta$. By Remark 2.1, we can choose two periodic orbits $\xi_{\alpha}$ and $\xi_{\beta}$ on $\mathcal{M}_{p_{0}, V_{0}}$ such that their rotation vectors are $\alpha q$ and $\beta q$ respectively.

Choose $x_{0} \in \mathbb{T}^{2}$ such that $\xi_{\alpha}$ and $\xi_{\beta}$ do not pass through $x_{0}$. Pick $\delta>0$ such that

$$
B_{2 \delta}\left(x_{0}\right) \cap \xi_{\alpha}(\mathbb{R})=\emptyset \quad \text { and } \quad B_{2 \delta}\left(x_{0}\right) \cap \xi_{\beta}(\mathbb{R})=\emptyset .
$$

See Figure 8 . Choose $\phi \in C^{\infty}\left(\mathbb{T}^{2}\right)$ satisfying

$$
\phi>0 \text { in } B_{\delta}\left(x_{0}\right) \text { and } \phi=0 \text { in } \mathbb{T}^{2} \backslash B_{\delta}\left(x_{0}\right) \text {. }
$$




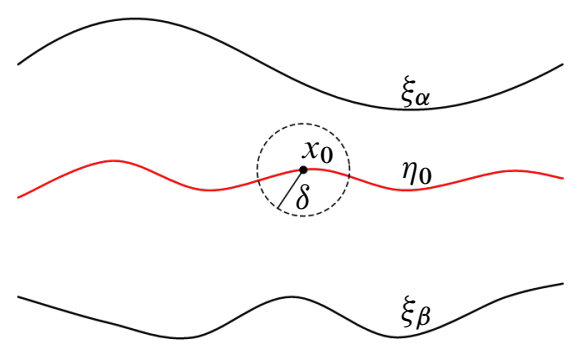

Figure 8

For $\delta>0$, denote

$$
V_{\delta}=V_{0}-\delta \phi
$$

It suffices to show that $V_{\delta} \in C(q, r)$. Suppose that $q \in \partial \bar{H}_{V_{\delta}}\left(p^{\prime}\right)$ for some $p^{\prime} \in \mathbb{R}^{2}$. The goal is to verify that

$$
\mathcal{M}_{p^{\prime}, V_{\delta}} \neq \mathbb{T}^{2} .
$$

Claim: $\bar{H}_{V_{\delta}}\left(p_{0}\right)=\bar{H}_{V_{0}}\left(p_{0}\right)$.

Since $V_{\delta} \leq V$, owing to the inf-max formula (1.4),

$$
\bar{H}_{V_{\delta}}\left(p_{0}\right) \leq \bar{H}_{V_{0}}\left(p_{0}\right) \text {. }
$$

So it suffices to show that

$$
\bar{H}_{V_{\delta}}\left(p_{0}\right) \geq \bar{H}_{V_{0}}\left(p_{0}\right)
$$

Due to Corollary 2.1,

$$
\begin{aligned}
\int_{0}^{T_{0}} \frac{1}{2}\left|\dot{\xi}_{\alpha}\right|^{2}-V_{\delta}\left(\xi_{\alpha}(t)\right)+\bar{H}_{V_{\delta}}\left(p_{0}\right) d t & \geq p_{0} \cdot(m, n) \\
& =\int_{0}^{T_{0}} \frac{1}{2}\left|\dot{\xi}_{\alpha}\right|^{2}-V_{0}\left(\xi_{\alpha}(t)\right)+\bar{H}_{V_{0}}\left(p_{0}\right) d t
\end{aligned}
$$

Hence $\bar{H}_{V_{\delta}}(p) \geq \bar{H}_{V_{0}}(p)$. Therefore our claim holds and $\xi_{\alpha}$ is also a periodic orbit on $\mathcal{M}_{p_{0}, V_{\delta}}$ and all periodic orbits on $\mathcal{M}_{p_{0}, V_{\delta}}$ have first homology class $(m, n)$. Similarly, $\xi_{\beta}$ is also a periodic orbit on $\mathcal{M}_{p_{0}, V_{\delta}}$. Hence

$$
q \in[\alpha q, \beta q] \subset \partial \bar{H}_{V_{\delta}}\left(p_{0}\right) .
$$

Therefore $\bar{H}_{V_{\delta}}$ is linear on $\left[p_{0}, p^{\prime}\right]$. Owing to Property 3 and (2.8),

$$
\mathcal{M}_{p_{0}, V_{\delta}}=\mathcal{M}_{p^{\prime}, V_{\delta}} .
$$

So we just need to prove that

$$
\mathcal{M}_{p_{0}, V_{\delta}} \neq \mathbb{T}^{2}
$$


We argue by contradiction. Assume that

$$
\mathcal{M}_{p_{0}, V_{\delta}}=\mathbb{T}^{2}
$$

Let $\eta_{0}:\left[0, \widetilde{T}_{0}\right] \rightarrow \mathbb{T}^{2}$ be the periodic orbit on $\mathcal{M}_{p_{0}, V_{\delta}}$ with $\eta_{0}(0)=x_{0}$. Here $\widetilde{T}_{0}>0$ is the minimum period. Then

$$
\begin{aligned}
p_{0} \cdot(m, n) & =\int_{0}^{\widetilde{T}} \frac{1}{2}\left|\dot{\eta}_{0}\right|^{2}-V_{\delta}\left(\eta_{0}\right)+\bar{H}_{V_{\delta}}\left(p_{0}\right) d t \\
& =\int_{0}^{\widetilde{T}} \frac{1}{2}\left|\dot{\eta}_{0}\right|^{2}-V_{\delta}\left(\eta_{0}\right)+\bar{H}_{V_{0}}\left(p_{0}\right) d t \\
& >\int_{0}^{\tilde{T}} \frac{1}{2}\left|\dot{\eta}_{0}\right|^{2}-V_{0}\left(\eta_{0}\right)+\bar{H}_{V_{0}}\left(p_{0}\right) d t .
\end{aligned}
$$

This contradicts Corollary 2.1.

The residual set $\mathcal{E}$ in Theorem 1.1 is chosen as

$$
\mathcal{E}=\bigcap_{q \in \mathbb{Q}^{2}, r \in \mathbb{Q}} C(q, r) .
$$

Here $\mathbb{Q}^{2}$ is the collection of points on $\mathbb{R}^{2}$ with both coordinates rational numbers.

Acknowledgments. The author would like to thank Jinxin Xue for extremely helpful discussions about Aubry-Mather theory and numerous suggestions to improve the presentation of this paper. The author first thought about this problem when he was visiting Nanjing University in China in 2019. Fruitful discussions with Chong-qing Cheng and Wei Cheng at Nanjing University and their hospitality are greatly appreciated. My gratitude also goes to Hongwei Gao for help drawing the figures in this paper. The author also would like to thank Professor Lawrence Craig Evans for his encouragement to write down this result. In addition, an anonymous referee is thanked for carefully reading the paper and providing helpful suggestions.

Funding. This work is partially supported by NSF grant DMS-2000191.

\section{References}

[1] V. Bangert, Mather sets for twist maps and geodesics on tori. In Dynamics reported, Vol. 1, pp. 1-56, Dynam. Report. Ser. Dynam. Systems Appl. 1, Wiley, Chichester, 1988 Zbl 0664.53021 MR 945963

[2] V. Bangert, Geodesic rays, Busemann functions and monotone twist maps. Calc. Var. Partial Differential Equations 2 (1994), no. 1, 49-63 Zbl 0794.58010 MR 1384394

[3] P. Bernard and G. Contreras, A generic property of families of Lagrangian systems. Ann. of Math. (2) 167 (2008), no. 3, 1099-1108 Zbl 1175.37067 MR 2415395 
[4] M. J. D. Carneiro, On minimizing measures of the action of autonomous Lagrangians. Nonlinearity 8 (1995), no. 6, 1077-1085 Zbl 0845.58023 MR 1363400

[5] C.-Q. Cheng and M. Zhou, Global normally hyperbolic invariant cylinders in Lagrangian systems. Math. Res. Lett. 23 (2016), no. 3, 685-705 Zbl 1381.37071 MR 3533191

[6] M. C. Concordel, Periodic homogenisation of Hamilton-Jacobi equations. II. Eikonal equations. Proc. Roy. Soc. Edinburgh Sect. A 127 (1997), no. 4, 665-689 Zbl 0883.35010 MR 1465414

[7] W. E, Aubry-Mather theory and periodic solutions of the forced Burgers equation. Comm. Pure Appl. Math. 52 (1999), no. 7, 811-828 Zbl 0916.35099 MR 1682812

[8] L. C. Evans and D. Gomes, Effective Hamiltonians and averaging for Hamiltonian dynamics. I. Arch. Ration. Mech. Anal. 157 (2001), no. 1, 1-33 Zbl 0986.37056 MR 1822413

[9] A. Fathi, The weak KAM theorem in Lagrangian dynamics, Cambridge University Press (2004).

[10] G. A. Hedlund, Geodesics on a two-dimensional Riemannian manifold with periodic coefficients. Ann. of Math. (2) 33 (1932), no. 4, 719-739 Zbl 58.1256.01 MR 1503086

[11] W. Jing, H. V. Tran, and Y. Yu, Effective fronts of polytope shapes. Minimax Theory Appl. 5 (2020), no. 2, 347-360 Zbl 1452.35021 MR 4132072

[12] P.-L. Lions, Generalized solutions of Hamilton-Jacobi equations. Res. Notes Math. 69, Pitman (Advanced Publishing Program), Boston, MA-London, 1982 Zbl 0497.35001 MR 667669

[13] P. L. Lions, G. C. Papanicolaou, and S. R. S. Varadhan, Homogenization of Hamilton-Jacobi equation. 1987, unpublished preprint

[14] J. N. Mather, Action minimizing invariant measures for positive definite Lagrangian systems. Math. Z. 207 (1991), no. 2, 169-207 Zbl 0696.58027 MR 1109661

[15] H. Mitake, H. V. Tran, and Y. Yu, Rate of convergence in periodic homogenization of Hamilton-Jacobi equations: the convex setting. Arch. Ration. Mech. Anal. 233 (2019), no. 2, 901-934 Zbl 1416.35034 MR 3951696

Received 7 October 2020; revised 26 February 2021; accepted 22 April 2021.

\section{Yifeng Yu}

Department of Mathematics, University of California, Irvine, 340 Rowland Hall, Irvine,

CA 92697-3875, USA; yifengy@uci.edu 